\title{
INTEGRAÇÃO ENTRE AS DIMENSÕES DA SUSTENTABILIDADE E A ATIVIDADE-FIM DAS IES BRASILEIRAS
}

\author{
Érika Pena Bedin ${ }^{1}$ \\ Luiz Carlos de Faria ${ }^{2}$
}

Resumo: Muitos estudos exploraram como as universidades podem aprimorar seu papel na sociedade, desenvolvendo novas abordagens e conteúdos educacionais e fornecendo apoio à pesquisa para objetivos de sustentabilidade. Porém, consideram a atividade-fim das IES quase sempre como estanque. Deste modo, o presente estudo visou integrar as dimensões da sustentabilidade com atividade-fim das IES brasileiras. Utilizou-se de métodos como revisão bibliográfica e documental para a análise de estudos científicos relacionados à indicadores de sustentabilidade em IES. Os resultados mostraram que embora existam constantes esforços tantos das IES públicas como privadas, a sustentabilidade ainda atinge baixos níveis de integração à atividade-fim.

Palavras-chave: Instituições de Ensino Superior (IES); Propósitos Fundamentais; Sustentabilidade; Dimensões e Práticas.

Abstract: Many studies have explored how universities can enhance their role in society by developing new educational approaches and content and providing research support for sustainability objectives. However, they almost always consider the purpose-activity of HEI as watertight. Thus, the present study aimed to integrate the dimensions of sustainability with the purpose-activity of Brazilian HEl. It used methods such as bibliographic and document review to analyze scientific studies related to sustainability indicators in HEI. The results showed that although there are constant efforts by both public and private HEI, sustainability still reaches low levels of integration with the end-activity.

Keywords: Higher Education Institutions (HEI); Fundamental Purposes; Sustainability; Dimensions and Practices.

\footnotetext{
1Universidade Federal de São Carlos - Campus Sorocaba. E-mail: erika@ufscar.br, Link para o Lattes: http://lattes.cnpq.br/4366016311242047

2Universidade Federal de São Carlos - Campus Sorocaba. E-mail: Icfaria@ufscar.br, Link para o Lattes: http://lattes.cnpq.br/2656343277991754
} 


\section{Introdução}

O debate sobre como as instituições podem se tornar mais sustentáveis cresceu exponencialmente ao longo do tempo, enfatizando oportunidades de inovação e competitividade (BANSAL, 2005; SHARMA E VREDENBURG, 1998). Com base em uma premissa diferente, outros estudos examinaram como as universidades podem integrar os conceitos de sustentabilidade como componentes de seus objetivos institucionais (BEYNAGHI et al., 2016).

$\mathrm{Na}$ Agenda 2030 das Nações Unidas para o Desenvolvimento Sustentável e sua definição dos Objetivos de Desenvolvimento Sustentável (ODS), a comunidade global expressa sua convicção de que os desafios globais só podem ser resolvidos trabalhando juntos. É de grande importância o estabelecimento de redes direcionadas de atores da política, negócios, ciência e sociedade civil. Cada vez mais, a própria comunidade científica, incluindo as universidades, está emergindo como principais atores organizacionais. No contexto da Década da Educação da UNESCO para o Desenvolvimento Sustentável, por exemplo, as universidades são consideradas centrais para a sustentabilidade. Em muitos aspectos, as universidades atuam como multiplicadoras da sustentabilidade; fazem recomendações importantes para a ação política sobre a regulamentação sustentável de certos setores industriais e desenvolvem inovações sustentáveis para e com empresas e sociedade. (STEPHENS et al. 2008).

Para Beynaghi et al. 2016, a análise qualitativa das tendências revela a crescente convergência dos desenvolvimentos no ensino superior e na sustentabilidade. Sendo tradicionalmente orientado para uma elite, a partir da década de 1950 o ensino superior se tornou um fenômeno de massa. Seguiuse uma crise de reputação e legitimidade, desencadeada por movimentos sociais na década de 1960. A década de 1970 testemunhou o nascimento do movimento ambiental, que se preocupava em limitar os impactos humanos no ambiente natural. Ao mesmo tempo, o ensino superior mudou à medida que as empresas industriais emergiam como um grupo-chave de partes interessadas, fornecendo fundos privados de terceiros para suprir as demandas financeiras da expansão e, simultaneamente, criando novas demandas para a instrumentalização das universidades.

Em termos de pesquisa, houve um aumento observável significativo na extensão e profundidade do trabalho científico universitário sobre sustentabilidade desde cerca de 2005 (FIGUEIRÓ; RAUFFLET, 2015). Segundo Karatzoglou (2013), o maior déficit nesta literatura (com base em um estudo de 123 publicações em periódicos) é que predominam estudos de caso específicos para universidades, produzindo achados prescritivos, mas até agora não há resultados empíricos generalizáveis.

Muitos estudos exploraram como as universidades podem aprimorar seu papel na sociedade, desenvolvendo novas abordagens e conteúdos educacionais e fornecendo apoio à pesquisa para objetivos de 
sustentabilidade. Em relação ao primeiro, as universidades são incentivadas a atualizar seus programas de treinamento para incluir tópicos relacionados à sustentabilidade em ofertas educacionais bem estabelecidas e novas, para abordar as competências agora exigidas pelo mercado. Mais especificamente, o investimento das empresas em sustentabilidade aumenta a necessidade de novos empregos relacionados ao meio ambiente, não necessariamente vistos no mercado de trabalho existente (OCDE, 2018).

Ao mesmo tempo, a crescente relevância da sustentabilidade para as funções comerciais existentes (por exemplo, marketing verde, design sustentável ou circularidade nas operações) significa que as competências profissionais devem ser atualizadas (RENWICK et al., 2013). Em relação ao apoio à pesquisa, as universidades podem se tornar importantes fornecedores de conhecimento relacionado à sustentabilidade para a indústria, no que é considerado um processo cada vez mais colaborativo (MUFF et al., 2013).

Pode-se observar muitos estudos sobre sustentabilidade, sustentabilidade no ensino superior e avaliação, medidas e classificação da sustentabilidade. No entanto, a maior parte ocorre em contextos regionais, nacionais ou locais. Ainda há relativamente pouco na literatura sobre classificações globais de sustentabilidade no ensino superior (THOMPSON, 1985; STUBBS; COCKLIN, 2008; RUSINKO, 2005; ROHWEDER, 2004) e, quando existente tende a relatar experiências e desafios enfrentados pelas IES na inclusão das questões ambientais em suas atividades de ensino, pesquisa, extensão e gestão universitária, considerando estas atividades quase sempre como estanque.

Deste modo, o presente estudo visa integrar as dimensões da sustentabilidade com a atividade-fim das IES brasileiras. Para este fim, o artigo teve como objetivos específicos: (1) discutir sobre o conceito e as dimensões de sustentabilidade utilizada para o estudo; (2) apresentar a atividade-fim das IES brasileiras e (3) integrar as dimensões da sustentabilidade à atividade-fim e gestão das IES.

\section{Metodologia}

A abordagem proposta para orientar a abordagem holística do estudo está estruturada em uma análise de estudos científicos relacionados à sustentabilidade nas IES e na sociedade como um todo (verificação cruzada, referência cruzada, conceitual e identificação de semelhanças) foi realizada levando em consideração iniciativas internacionais que promovem a sustentabilidade na educação e na sociedade, estruturas de avaliação existentes para a sustentabilidade e publicações internacionais (referências normativas, relatórios e artigos revisados por pares), que fundamentaram as reflexões críticas dos autores sobre sustentabilidade nas IES.

A revisão sistemática de literatura proporciona a investigação de diferenças e similaridades (ASHBY et al., 2012). Nesse contexto, a revisão 
sistemática de literatura foi utilizada para explorar sobre a trajetória da sustentabilidade em IES ao longo dos anos. Devido ao escopo estendido e diversificado de sua aplicação potencial, essa pesquisa visou identificar artigos publicados, com relevância e abrangência. Os documentos foram identificados a partir da base de dados Web of Science (WoS). Tal base de dados oferece uma ampla cobertura de disciplinas consideradas relevantes, bem como acesso a dados bibliográficos para posterior elaboração de tabelas e gráficos.

Os termos de pesquisa foram identificados e refinou-se ainda mais os critérios de seleção revisando uma amostra aleatória de publicações dos resultados da pesquisa. Uma pesquisa abrangente foi realizada, empregando a combinação final de termos de pesquisa, "Sustainability" AND "University". Em relação ao período temporal, optou-se pela não restrição, a fim de garantir uma maior amplitude da revisão sistemática. Com isso, o levantamento foi realizado entre 1970 e 2019.

A análise documental foi realizada examinando-se documentos oficiais como sites e banco de dados abertos das IES, do Ministério da Educação (MEC) e da Coordenação de Aperfeiçoamento de Pessoal de Nível Superior (CAPES). Foram coletados dados das IES dos 27 estados brasileiros, abrangendo a totalidade de população das 2.608 IES brasileiras, sem dados perdidos.

Tais preceitos metodológicos refletem uma visão holística da sustentabilidade nas IES e um alinhamento às recomendações transmitidas por organizações e publicações internacionais.

\section{Conceito e dimensões da sustentabilidade}

Embora amplamente abordados na literatura científica, os termos sustentável, sustentabilidade e desenvolvimento sustentável, podem ter interpretações diferentes dependendo do autor ou da área em questão. Deste modo, para o estudo de sustentabilidade e desenvolvimento sustentável são tratados como sinônimos, sendo este último àquele que atende "as necessidades atuais sem comprometer a habilidade das futuras gerações de atender suas próprias necessidades" (ONU, 1987).

Porém, a aplicação de tal conceito traz dificuldades reais, uma vez que a satisfação das necessidades atuais, como a busca por energia, está resultando em níveis de degradação ambiental nunca vistos antes. Por outro lado, o exercício em se estimar as necessidades de gerações futuras não é algo fácil, uma vez que a evolução das sociedades é dinâmica. Além disso, mesmo que consigamos estimar tais necessidades com certa eficiência, a história mostra que o desenvolvimento dentro das gerações quase sempre resulta em ações em que as gerações futuras ficam desassistidas, se consideramos um intervalo de tempo maior. Por exemplo, foi estimada por gerações anteriores que a necessidade atual seria de alimentos, porém dados da Organização das Nações Unidas (ONU) revelam que, por ano, 
aproximadamente um terço dos alimentos produzidos em todo o mundo não é consumido pela população, sendo perdido em alguma etapa da cadeia de produção ou desperdiçado no elo final. Isso sem contar que há uma série de desperdícios embutidos que agravam ainda mais o cenário global. A cadeia de produção e distribuição de alimentos necessita de água, terra, energia e combustíveis. O alimento que vai para o lixo enterra junto com ele todos esses recursos que foram consumidos durante o seu processo de produção e causa impactos nas três dimensões da sustentabilidade, social, econômica e ambiental.

A crise ambiental atual é resultado, por um lado, pelo esgotamento e degradação de muitos recursos naturais e, por outro, da deposição de substâncias não desejadas pela economia (resíduos). Ela veio questionar a racionalidade e as teorias que impulsionaram e legitimaram o crescimento econômico anteriormente previsto. A sustentabilidade aparece como um critério normativo para a reconstrução da ordem econômica, como uma condição para a sobrevivência humana e um suporte para chegar a um desenvolvimento duradouro, questionando as próprias bases de produção.

Visando a integração da sustentabilidade como um todo, procurou-se identificar as dimensões de sustentabilidade abordadas na literatura. Foram identificadas três dimensões: econômica, social e ambiental (WAAS et al., 2011; GODEMANN et al., 2014; AMARAL et al., 2015; SAMMALISTO et al., 2015). No entanto, é cada vez mais comum encontrar outros pilares, principalmente institucionais (LOZANO, 2008; DISTERHEFT et al., 2013; LEAL FILHO et al., 2015) e culturais (LOZANO, 2008; DISTERHEFT et al., 2013; LEAL FILHO et al. 2015). Além disso, quatro dimensões também foram propostas para práticas de sustentabilidade e a implementação do desenvolvimento sustentável nas IES e que serão adotados neste estudo: ambiental, econômica, social e institucional (LOZANO, 2011; ALONSOALMEIDA et al., 2015; LARRÁN JORGE et al., 2015; ALEIXO et al., 2016). O Quadro 1 resume as principais dimensões práticas da sustentabilidade nas IES.

Quadro 1: Dimensões e práticas da sustentabilidade nas IES

\begin{tabular}{|c|l|}
\hline Dimensões & \multicolumn{1}{|c|}{ Práticas } \\
\hline Ambiental & $\begin{array}{l}\text { Declarações e ações relacionadas ao envolvimento das IES em questões } \\
\text { ambientais e escassez de recursos (meio ambiente e gestão de recursos } \\
\text { naturais; prevenção da poluição; proteção do meio ambiente e da biodiversidade; } \\
\text { restauração de habitats naturais; pegada ecológica; recursos não renováveis; } \\
\text { esgotamento de materiais; degradação). }\end{array}$ \\
\hline Econômica & $\begin{array}{l}\text { Declarações e ações relacionadas ao impacto econômico direto e e } \\
\text { sustentabilidade financeira das IES (situação financeira; resultados; eficiência). }\end{array}$ \\
\hline
\end{tabular}

Continua... 
...continuação.

\begin{tabular}{|c|l|}
\hline Social & $\begin{array}{l}\text { Declarações e explicações sobre políticas e procedimentos relativos a direitos } \\
\text { humanos (práticas trabalhistas; direitos humanos; qualidade de vida, saúde e } \\
\text { segurança ocupacional; dimensão da equidade; treinamento de funcionários, } \\
\text { envolvimento em questões sociais e ação na comunidade das IES). }\end{array}$ \\
\hline Institucional & $\begin{array}{l}\text { Declarações sobre pontos de vista, valores, estratégia, transparência na } \\
\text { governança, compromissos éticos das IES e cartas e parcerias sobre critérios } \\
\text { nacionais e internacionais para promover o desenvolvimento } \\
\text { sustentável. Também foram consideradas práticas em educação, pesquisa, } \\
\text { operações universitárias (por exemplo, certificações), divulgação e avaliação da } \\
\text { comunidade e relatórios. }\end{array}$ \\
\hline
\end{tabular}

Fonte: Adaptado de Aleixo et al. (2016)

\section{Atividade-fim das IES brasileiras}

A Constituição Federal de 1988, em seu artigo 207, estabelece como propósito fundamental a atividade-fim das IES brasileiras, por meio do princípio da indissociabilidade entre ensino, pesquisa e extensão (QUADRO 2).

Quadro 2: Atividade-fim das IES brasileiras

\begin{tabular}{|c|l|}
\hline Atividade & \multicolumn{1}{|c|}{ Práticas } \\
\hline Ensino & $\begin{array}{l}\text { Práticas voltadas ao aprendizado dos discentes para formação profissional, como } \\
\text { as horas destinadas às aulas em sala, laboratórios, atividades de monitoria, dentre } \\
\text { outras. }\end{array}$ \\
\hline Pesquisa & $\begin{array}{l}\text { Ações desenvolvidas com o objetivo de fomentar as atividades de pesquisa dentro } \\
\text { das IES. Geralmente acontecem nas pós-graduações, principalmente do tipo stricto- } \\
\text { sensu, por meio de iniciação científica ou projetos. }\end{array}$ \\
\hline Extensão & $\begin{array}{l}\text { Ações que estendem o conhecimento à sociedade como um todo, buscando colher } \\
\text { informações e demandas que ajudem a IES a se envolver com a realidade à sua } \\
\text { volta. visam à difusão das conquistas e benefícios resultantes da criação cultural e } \\
\text { da pesquisa científica e tecnológica geradas, criando um elo entre a sociedade e a } \\
\text { IES. }\end{array}$ \\
\hline
\end{tabular}

Fonte: Dados da pesquisa

De maneira explanada, o ensino é a transmissão de conhecimentos através da abstração e, quando possível, prática de determinados assuntos, feitas por um docente. É aquele que desencadeia conhecimento capaz de transformar a atuação do indivíduo como ser social, convergindo e articulando de maneira equilibrada entre as dimensões científica, investigativa e pedagógica. Já a pesquisa é a oportunidade de aplicar e/ou desenvolver novos conceitos a partir das bases construídas pela etapa do ensino. É o processo de materialização do saber a partir da produção de novos conhecimentos baseado de problemas emergentes da prática social. Por fim, a extensão é a aplicação direta do conhecimento obtido nas fases do ensino e pesquisa na sociedade. É rica em aprendizados acadêmicos e sociais, o que contribui para a formação de 
um profissional mais integrado com as demandas da sociedade (BRASIL, 1988).

Porém, a concretização de tal indissociabilidade entre ensino, pesquisa e extensão na prática acadêmica, de fato, tem se mostrado difícil, pois o que se observa é que, via de regra, o trabalho continua fragmentado entre ensinar, pesquisar e fazer extensão. Em suma maioria, isso se deve ao fato do questionamento da sociedade às IES quanto a utilidade do conhecimento e da formação nelas produzidos, demandando e atribuindo novas funções. Por exemplo, o ensino costuma ser avaliado pelo Ministério da Educação (MEC), por meio do Índice Geral de Cursos (IGC), que sintetiza em um único indicador a qualidade dos cursos, avaliando o corpo discente, docente e a infraestrutura das IES.

Além disso, as atividades de pesquisa, que culminam em publicações, acabam sendo mais valorizadas, por serem utilizadas na maioria dos critérios de avaliação dos programas de pós-graduação, por meio do conceito Coordenação de Aperfeiçoamento de Pessoal de Nível Superior (CAPES), que avalia a qualidade dos cursos de pós-graduação stricto sensu e por órgãos fomentadores de financiamentos, impactando inclusive na pontuação da progressão funcional dos docentes em IES públicas.

Soma-se a isso, de um lado, reconfigurações do poder público que implicam em diminuição de recursos para financiamento para as IES públicas, enquanto de outro lado as IES com financiamento privado contribuem para uma hierarquização interna de áreas e de conhecimentos, conforme sua maior ou menor facilidade de captar recursos. Tal panorama fortalece tensões internas às IES, que historicamente é consolidada por meio de uma estrutura rígida e formal sobre seu papel de produtora e detentora do conhecimento.

Estas situações evidenciam que a associação entre as funções de ensino, pesquisa e extensão é tarefa institucional, não sendo realizada apenas pelo professor ou pelo estudante. Há demanda de uma estrutura organizacional voltada para a superação da divisão que marca o modelo usualmente adotado. Nesse sentido, ainda há muito a caminhar, pois, se atualmente a presença formal do ensino, da pesquisa e da extensão são garantidas como princípio fundamental e constitucional da missão da Universidade, a coexistência delas não significa, necessariamente, que elas sejam indissociáveis.

Um indicador que reforça tal argumento é a consideração do ensino, pesquisa e extensão como atividades distintas, que deveriam ser desenvolvidas indissociavelmente. Atualmente, vários estudantes ainda são formados sem sequer vivenciarem atividades de pesquisa, nem elementos intrínsecos à extensão. Pior, a possibilidade de que participem é colocada de maneira formal, seja por meio de atividades formativas, bolsas ou atividades de iniciação científica. Porém, não deveria ser somente possível, mas obrigatória, fornecendo condições para isso, conforme o princípio constitucional, de maneira a dialogar com outros saberes e com a interdisciplinaridade. 
Além da atividade-fim: ensino, pesquisa e extensão, se faz necessário adicionar a gestão das IES (CORTESE, 2003, LOZANO, 2006, LOZANO, 2011, LOZANO et al., 2013a, LOZANO et al., 2013b). Tal atividade envolve a comunicação das IES com as diferentes partes interessadas por meio do ensino, pesquisa, operações no campus, sensibilização da comunidade e conscientização da comunidade (por exemplo, avaliação e relatórios da sustentabilidade).

\section{Resultados e Discussões}

Esta seção busca integrar as dimensões da sustentabilidade, à atividade-fim e à gestão das IES brasileiras.

\section{Integração entre sustentabilidade, atividade-fim e gestão das IES brasileiras}

As atividades relacionadas ao ensino devem incluir a revisão dos resultados da aprendizagem e a reformulação do currículo (DISTERHEFT et al., 2016) e a introdução de conceitos de sustentabilidade como um assunto no currículo de todas as disciplinas e cursos das instituições de ensino superior, bem como oficinas, conferências e seminários. A integração da sustentabilidade nos currículos pode ser feita verticalmente (sustentabilidade integrada por meio de cursos específicos relacionados à sustentabilidade) ou horizontalmente (sustentabilidade integrada em diferentes cursos regulares do currículo) (STOUGH et al., 2017).

As atividades de pesquisa incentivam a análise sobre questões de sustentabilidade que abordam os desafios da sociedade, bem como grupos de pesquisa interdisciplinares para uma nova abordagem de maneira sustentável (POPESCU; BELEAU, 2014).

O alcance da extensão abrange atividades nas quais as instituições de ensino superior estão envolvidas no desenvolvimento regional e local e com a sociedade civil para promover um ambiente mais habitável, socialmente inclusivo e eficiente em termos de recursos (POPESCU; BELEAU, 2014). No entanto, como observam Dyer e Dyer (2017), ensino, pesquisa e prática interdisciplinares são necessários para promoção da sustentabilidade na sociedade.

As atividades de gestão nos campi estão relacionadas a iniciativas e campanhas de campus verde, com foco em melhorias operacionais (DISTERHEFT et al., 2016). Porém, enquanto na gestão de IES privada há a disponibilidade financeira e estrutural para contratação de recursos pessoais para a realização do gerenciamento, na gestão de IES pública a gestão ocorre pela própria comunidade acadêmica (técnico-administrativos e docentes), com os mesmos recursos básicos, repassados para seu funcionamento. O Quadro 3 apresenta alguns estudos nacionais se destacam no quesito gestão ambiental. 
Quadro 3: Principais artigos sobre IES brasileiras

\begin{tabular}{|c|c|c|c|}
\hline Autores & Artigo & Foco & Resultados \\
\hline $\begin{array}{l}\text { Boff; Oro e } \\
\text { Beuren } \\
\text { (2008) }\end{array}$ & $\begin{array}{l}\text { Gestão } \\
\text { ambiental em } \\
\text { Instituição de } \\
\text { Ensino } \\
\text { Superior na } \\
\text { visão de seus } \\
\text { dirigentes. }\end{array}$ & $\begin{array}{l}\text { Gestão } \\
\text { ambiental de } \\
\text { uma IES em } \\
\text { Santa Catarina. }\end{array}$ & $\begin{array}{l}\text { Incentivo de conscientização da quantia } \\
\text { de lixo produzida individualmente e dos } \\
\text { danos causados à natureza, por meio } \\
\text { de Educação Ambiental, iniciando pela } \\
\text { importância dos recursos do meio } \\
\text { ambiente. }\end{array}$ \\
\hline $\begin{array}{l}\text { Bolzan; } \\
\text { Weber e } \\
\text { Löbler } \\
(2010)\end{array}$ & $\begin{array}{l}\text { Alinhamento } \\
\text { ambiental em } \\
\text { uma } \\
\text { Instituição } \\
\text { Pública de } \\
\text { Ensino } \\
\text { Superior. }\end{array}$ & $\begin{array}{l}\text { Tratamento de } \\
\text { resíduos } \\
\text { sólidos em uma } \\
\text { IES. }\end{array}$ & $\begin{array}{l}\text { Construção de um mapeamento dos } \\
\text { resíduos gerados na IES e identificação } \\
\text { dos principais resíduos, papel e copo } \\
\text { plástico, provenientes de rotinas } \\
\text { administrativas e pedagógicas, que } \\
\text { suscitavam tratamento adequado. }\end{array}$ \\
\hline $\begin{array}{l}\text { Dziedzic e } \\
\text { Dziedzic } \\
(2010)\end{array}$ & $\begin{array}{l}\text { Diagnóstico e } \\
\text { proposta de } \\
\text { redução de } \\
\text { emissões - } \\
\text { Campus } \\
\text { Universidade } \\
\text { Positivo. }\end{array}$ & $\begin{array}{l}\text { Fluxo de } \\
\text { materiais em } \\
\text { uma IES em } \\
\text { Curitiba, } \\
\text { focando na } \\
\text { minimização de } \\
\text { emissões, } \\
\text { consumo de } \\
\text { água e energia } \\
\text { e produção de } \\
\text { esgoto e } \\
\text { resíduos. }\end{array}$ & $\begin{array}{l}\text { Criação de planilha para o diagnóstico } \\
\text { do fluxo de materiais a partir do cálculo } \\
\text { de emissões, que incluem modificações } \\
\text { em válvulas de descarga, captação de } \\
\text { água da chuva, tratamento de esgoto } \\
\text { por banhado construído, produção de } \\
\text { energia a partir do biogás dos resíduos, } \\
\text { instalação de painéis fotovoltaicos e } \\
\text { alterações na eficiência de } \\
\text { equipamentos ou mudanças no padrão } \\
\text { de consumo. Para cada mudança, } \\
\text { também foram efetuados estudos de } \\
\text { viabilidade por custo x benefício e } \\
\text { redução de emissões. Finalmente, foi } \\
\text { realizada uma proposta de } \\
\text { modificações ao campus, com base nos } \\
\text { aspectos ecológicos e econômicos. }\end{array}$ \\
\hline $\begin{array}{l}\text { Lima Jr; } \\
\text { Oiko e } \\
\text { Cavicchioli } \\
(2010)\end{array}$ & $\begin{array}{l}\text { Gestão de } \\
\text { resíduos em } \\
\text { universidade: } \\
\text { caracterização } \\
\text { e análise da } \\
\text { destinação de } \\
\text { resíduos } \\
\text { sólidos. }\end{array}$ & $\begin{array}{l}\text { Caracterização, } \\
\text { destinação final } \\
\text { de resíduos } \\
\text { sólidos e } \\
\text { avaliação do } \\
\text { nível de } \\
\text { conscientização } \\
\text { dos } \\
\text { funcionários da } \\
\text { Universidade } \\
\text { Estadual de } \\
\text { Maringá (UEM). }\end{array}$ & $\begin{array}{l}\text { Apresentação de propostas adequadas } \\
\text { ao atual panorama da cadeia de } \\
\text { resíduos da UEM, sendo que algumas } \\
\text { foram desenvolvidas com base em } \\
\text { algumas práticas de gerenciamento de } \\
\text { resíduos implantadas em outras } \\
\text { universidades. }\end{array}$ \\
\hline
\end{tabular}

Fonte: Dados da pesquisa

De acordo com os estudos acima, percebe-se que as IES tomaram consciência dos impactos que sua atividade exerce sobre o meio ambiente e tais preocupações ambientais funcionaram como força motriz para a 
sustentabilidade. Isso porque, como afirma Larrán Jorge et al. (2015), os líderes das IES devem formalizar seu compromisso com a sustentabilidade assinando declarações.

Para White (2013), a sustentabilidade das IES implica a adoção de objetivos e indicadores mensuráveis e gerenciáveis. As ferramentas de avaliação da sustentabilidade (por exemplo, AASHE - A Associação para o Progresso da Sustentabilidade no Ensino Superior) podem desempenhar um papel estratégico não apenas no desenvolvimento de uma abordagem holística e sistêmica da sustentabilidade, mas também como um facilitador vital para a mudança em direção à sustentabilidade. Existem inúmeras ferramentas de avaliação de sustentabilidade para o ensino superior, e uma extensa revisão da literatura sobre isso pode ser encontrada em Larrán Jorge et al. (2015).

Popescu e Beleau (2014) observam que não existe um único caminho ou instrumento de uso geral para implementar os valores de sustentabilidade ou avaliar seus resultados. Eles argumentam, portanto, que a elaboração de modelos unitários poderia ajudar a melhorar a eficácia da abordagem universitária e controlar a implementação dos programas desenvolvidos nos níveis internacional, regional e nacional.

Nos últimos anos, tem havido crescente discussão sobre a importância e as contribuições dos rankings das IES (e como isso pode ser um parâmetro/vantagem distintivo para as instituições) (SHIN; TOUTKOUSHIAN, 2011; MOURA; MOURA, 2013). Os rankings são uma forma de demonstrar o compromisso das instituições com ações voltadas para a sustentabilidade, ou mesmo motivar internamente sobre a necessidade e importância de tais ações. Entretanto, por serem de adesão voluntária as informações são autodeclaradas, e não necessariamente acompanhadas de evidências documentais, ou outras, que permitam avaliar sua veracidade ou mesmo a efetividade das ações ditas sustentáveis.

Outra forma possível das IES demonstrarem seus compromissos com a sustentabilidade é por meio de certificações voluntárias. Já existem vários processos formais em nível nacional e internacional para os mais variados contextos, como o do Forest Stewardship Council (FSC) e da Certificação Florestal (CERFLOR) para produtos oriundos de florestas. Vale destacar que as certificações voluntárias, diferentemente de normas compulsórias, não estão no âmbito de fiscalização dos órgãos públicos, embora o cumprimento de normas compulsórias sejam requisitos de muitos sistemas de certificação, como o FSC CERFLOR. Por isso a credibilidade desses sistemas perante os consumidores dos produtos ou serviços das instituições certificadas é um elemento essencial. Por isso, dentre outros elementos, o processo de certificação é, por definição, conduzido por uma organização independente tanto do "controlador" do sistema de certificação, quanto da instituição candidata a certificação (INMETRO, 2021a). No Brasil, as organizações que conduzem as auditorias de certificação devem, ainda, ser acreditadas pelo Instituto Nacional de Metrologia, Qualidade e Tecnologia (INMETRO), que 
reconhece sua competência para avaliar, com base em regras preestabelecidas para executar a avaliação da conformidade.

No contexto da sustentabilidade, a certificação mais conhecida mundialmente talvez seja a de Sistemas de Gestão Ambiental (SGA) da International Organization for Standardization (ISO), a ISO 14001. A certificação ISO 14001 provê um modelo básico de estabelecimento de um Sistema de Gestão Ambiental (SGA), um conjunto de processos de gerenciamento que requer das empresas a identificação, a mensuração e 0 controle de seus impactos ambientais no meio ambiente em que operam, incluindo aspectos relacionados a ar, água, solo, flora, fauna e seres humanos (BANSAL, 2005).

A ISO 14001 não restringe seu escopo, ela é aplicável tanto para instituições privadas como para as públicas, embora não seja comum. Ao consultar as certificações válidas de IES brasileiras (INMETRO, 2021b), apenas duas estão certificadas pelo ISO 14001, ambas de categoria privada: a Universidade Positivo e a Universidade do Vale do Rio dos Sinos (UNISINOS).

Isso talvez se deva, em grande parte, devido à necessidade em demonstrar os benefícios resultantes de um SGA, como reestruturação na organização, reestabelecimento de prioridades organizacionais e conscientização do SGA por todos os envolvidos. Também requer um nível de conscientização e motivação entre, pelo menos, a maioria das outras partes interessadas, dentro e fora da organização, para a aplicação bem-sucedida do SGA e para sua melhoria contínua. O senso de realização de uma organização para entregar melhor desempenho ambiental também precisa ser gerado ao fornecer retorno e apoio para estimular o sentimento de que as pessoas realmente podem (e fazem) a diferença, ao mesmo tempo incentivando outras boas ideias.

Na mesma lógica das certificações, no âmbito dos entes públicos das esferas federal, estadual e municipal, e nos poderes executivo, legislativo e judiciário, destaca-se atualmente o programa federal, de adesão voluntária, criado pelo Ministério do Meio Ambiente (MMA) denominado de Agenda Ambiental na Administração Pública (A3P). O programa destina-se a estimular os gestores públicos a incorporar princípios e critérios de gestão ambiental em suas atividades rotineiras. A A3P é baseada em seis eixos temáticos e visa reduzir gastos institucionais por meio do uso racional dos recursos naturais $\mathrm{e}$ dos bens públicos, da gestão adequada dos resíduos, e de outros princípios, tendo como foco a mudança de comportamento (MMA, 2017). O autor destaca que a redução de gastos é consequência de uma gestão socioambiental, e não o objetivo do programa final.

Diferentemente da ISO14001, a A3P, criada no final de 1999 pelo MMA, não é um sistema de certificação propriamente dito, porque the carece elementos essênciais desse processo, como as auditorias de terceira parte como garantia de maior credibilidade externa. A semelhança da ISO 14001 como resposta do setor privado à pressão social, a A3P foi a alternativa do

revista brasileira educação ambiental 
governo brasileiro para o setor público. As IES que atualmente aderiram à A3P são apresentadas na Quadro 4.

Quadro 4: IES com adesão à A3P

\begin{tabular}{|c|c|}
\hline IES & UF \\
\hline Centro Federal de Educação Tecnológica Celso Suckow da Fonseca (CEFET) & RJ \\
\hline Escola de Gestão Pública de Estado do Ceará (EGPCE) & CE \\
\hline Escola Nacional de Administração Pública (ENAP) & DF \\
\hline Escola Superior do Ministério Público da União (ESMPU) & DF \\
\hline Fundação Universidade Federal do Vale do São Francisco (UNIVASF) & $\mathrm{PE}$ \\
\hline $\begin{array}{l}\text { Instituto Federal de Educação, Ciência e Tecnologia Catarinense (IFSC - Campus } \\
\text { Brusque) }\end{array}$ & SC \\
\hline Instituto Federal de Educação, Ciência e Tecnologia de Brasília (IFB) & DF \\
\hline Instituto Federal de Educação, Ciência e Tecnologia de Santa Catarina (IFSC) & SC \\
\hline Instituto Federal de Educação, Ciência e Tecnologia do Acre (IFAC) & $\mathrm{AC}$ \\
\hline Instituto Federal de Educação, Ciência e Tecnologia do Amazonas (IFAM) & AM \\
\hline $\begin{array}{l}\text { Instituto Federal de Educação, Ciência e Tecnologia do Espírito Santo (IFES - Campus } \\
\text { Guarapari) }\end{array}$ & ES \\
\hline Instituto Federal de Educação, Ciência e Tecnologia do Piauí (IFPI - Campus Corrente) & $\mathrm{PI}$ \\
\hline Instituto Federal de Educação, Ciência e Tecnologia do Piauí (IFPI - Campus Floriano) & $\mathrm{PI}$ \\
\hline Instituto Federal de Educação, Ciência e Tecnologia do Rio de Janeiro (IFRJ) & RJ \\
\hline $\begin{array}{l}\text { Instituto Federal de Educação, Ciência e Tecnologia do Sul de Minas Gerais } \\
\text { (IFSULDEMINAS) }\end{array}$ & MG \\
\hline Instituto Federal de Educação, Ciência e Tecnologia Goiano (IFGO) & GO \\
\hline Universidade Federal do Maranhão (UFMA) & MA \\
\hline Universidade Federal da Paraíba (UFPB) & PB \\
\hline Universidade Federal de Alfenas (UNIFAL) & $\mathrm{AL}$ \\
\hline Universidade Federal de Grande Dourados (UFGD) & MS \\
\hline Universidade Federal de Pernambuco (UFPE - Campus do Agreste) & PE \\
\hline Universidade Federal de Santa Catarina (UFSC) & SC \\
\hline Universidade Federal de Santa Maria (UFSM - Campus Palmeira das Missões) & RS \\
\hline Universidade Federal de Sergipe (UFS) & SE \\
\hline Universidade Federal de Uberlândia (UFU) & MG \\
\hline Universidade Federal do Cariri (UFCA) & CE \\
\hline Universidade Federal do Rio Grande (UFRG) & RS \\
\hline Universidade Federal Rural da Amazônia (U & $\mathrm{PA}$ \\
\hline
\end{tabular}

Fonte: Dados da pesquisa

Revbea, São Paulo, V. 16, № 6: 83-103, 2021. 
Enquanto apenas duas IES privadas são certificadas pela ISO 14001, 28 públicas aderiram à A3P. Segundo o MMA (2017), a A3P se encontra em harmonia com o princípio da economicidade, que se traduz na relação custobenefício e, ao mesmo tempo, atende ao princípio constitucional da eficiência na administração pública. Além disso, pode ser considerada como o marco indutor de adoção da gestão socioambiental no âmbito da administração pública brasileira. Segundo o autor, busca-se, com a implementação da A3P, uma ação exemplar do gestor público a partir da compreensão do que é a responsabilidade socioambiental que tem como foco a mudança de comportamento.

Reitera-se, oportunamente, que A3P não se trata de uma certificação ambiental formal. Embora em seu processo seja exigido compromisso formal dos entes públicos, especialmente o termo de adesão voluntário, porém as informações sobre o desempenho ambiental são disponibilizadas pelo próprio órgão que adere ao programa, não havendo nenhuma checagem posterior quanto à sua veracidade, compreendendo apenas evidências documentais. Além disso, alguns indicadores e critérios importantes para as IES podem não ter sido suficientemente incluídos ou claramente descritos. Apesar de todas as limitações descritas sobre os rankings e programas socioambientais governamentais, eles ofereçam oportunidade para as IES em demonstrar seus compromissos rumo à sustentabilidade.

De forma geral as IES podem contribuir de forma relevante para a indução da sustentabilidade na sociedade de formas principais: no desenvolvendo e disseminando técnicas e atitudes mais sustentáveis por meio da execução de suas atividades-fim de ensino, pesquisa e extensão; e por meio da adequada gestão de seus próprios espaços territoriais de atuação, servindo assim de exemplo para outros entes, setores e atividades.

Nesse contexto, a fim de propor as relações entre as atividades-fim das IES (gestão, ensino, pesquisa e extensão) com os preceitos da sustentabilidade se faz necessário identificar indicadores. Uma abordagem eficaz para isso é a da SMART (Specific, Measurable, Attainable, Relevant e Time-bound), que define cinco critérios que podem ser aplicados para definir indicadores, eles precisam ser: específicos, mensuráveis, atingíveis, relevantes e abranger um limite de tempo (SHAHIN; MAHBOD, 2007).

Com o intuito de identificar indicadores de sustentabilidade para IES brasileiras, foram operacionalizados nove construtos a fim de integrar as dimensões da sustentabilidade à atividade-fim e gestão das IES: Políticas de Sustentabilidade (PS), Conscientização e Participação (CP), Responsabilidade Socioambiental (RS), Docência (DO), Energia (EN), Água (AG), Mobilidade (MO), Resíduos (RE) e Contratação Responsável (CR); por meio de vinte e sete variáveis que atendem a abordagem SMART (PS1, PS2, PS3, CP1, CP2, CP3, RS1, RS2, RS3, DO1, DO1, DO3, EN1, EN2, EN3, AG1, AG2, AG3, MO1, MO2, MO3, RE1, RE2, RE3, CR1, CR2, CR3), conforme apresentado no Quadro 5. 
Quadro 5: Operacionalização dos construtos

\begin{tabular}{|c|c|}
\hline Construto & Operacionalização \\
\hline $\begin{array}{c}\text { Políticas de } \\
\text { Sustentabilidade } \\
\text { (PS) }\end{array}$ & $\begin{array}{l}\text { PS1: Existe um documento que define a política que a universidade } \\
\text { pretende desenvolver no âmbito da sustentabilidade/ambiente. } \\
\text { PS2: A ação ambiental e/ou pela sustentabilidade corresponde a um plano } \\
\text { de ação ambiental ou sistema integral de sustentabilidade. } \\
\text { PS3: Dentro do plano de ação ambiental/sustentabilidade existe um } \\
\text { objetivo ou sistema para avaliar o impacto ambiental gerado pela } \\
\text { universidade. }\end{array}$ \\
\hline $\begin{array}{c}\text { Conscientização } \\
\text { e Participação } \\
\text { (CP) }\end{array}$ & $\begin{array}{l}\text { CP1: São geralmente realizadas pesquisas sobre sustentabilidade em geral } \\
\text { para a comunidade universitária. } \\
\text { CP2: São realizadas atividades extracurriculares de sensibilização e } \\
\text { conscientização sobre temas de ambiente e sustentabilidade. } \\
\text { CP3: Existem incentivos monetários/acadêmicos e servidores que se } \\
\text { dedicam a atividades de sustentabilidade/meio ambiente. }\end{array}$ \\
\hline $\begin{array}{c}\text { Responsabilidade } \\
\text { Socioambiental } \\
\text { (RS) }\end{array}$ & $\begin{array}{l}\text { RS1: Existe um plano específico, eixo estratégico ou plano de ação de } \\
\text { responsabilidade social aprovado por uma autoridade ou órgão da } \\
\text { universidade } \\
\text { RS2: Existe um órgão consultivo no qual estão representados os diferentes } \\
\text { grupos da comunidade universitária e cuja missão seja a avaliação e/ou o } \\
\text { monitoramento das atividades de responsabilidade social. } \\
\text { RS3: Existe uma estratégia de comunicação do plano de } \\
\text { sustentabilidade/ambiente à comunidade universitária e agentes externos. }\end{array}$ \\
\hline $\begin{array}{c}\text { Docência } \\
\text { (DO) }\end{array}$ & $\begin{array}{l}\text { D01: Existência na política de sustentabilidade menção expressa das } \\
\text { atividades docentes. } \\
\text { DO2: Incorporação de pelo menos } 10 \% \text { dos currículos acadêmicos das } \\
\text { diferentes carreiras na perspectiva da sustentabilidade. } \\
\text { DO3: Realização de projetos de conclusão de curso ou de pós-graduação } \\
\text { relacionados à sustentabilidade na universidade. }\end{array}$ \\
\hline Energia & $\begin{array}{l}\text { EN1: Existência de um sistema de controle de consumo de energia com } \\
\text { medidores independentes nos edifícios no campus. } \\
\text { EN2: Realização de diagnósticos e/ou auditorias energéticas nos edifícios } \\
\text { (monitoramento de consumo, análise e revisão das potências contratadas, } \\
\text { análises dos hábitos de consumo dos usuários e das propostas de } \\
\text { atuação). } \\
\text { EN3: Desenvolvimento de planos e/ou medidas para reduzir o consumo em } \\
\text { iluminação no interior e no exterior dos edifícios (luminárias de baixo } \\
\text { consumo, detectores de presença, etc.). }\end{array}$ \\
\hline $\begin{array}{l}\text { Água } \\
\text { (AG) }\end{array}$ & $\begin{array}{l}\text { AG1: Existência de um sistema de controle de consumo de água com } \\
\text { medidores independentes nos edifícios no campus. } \\
\text { AG2: Existência/elaboração de um plano específico, eixo estratégico ou } \\
\text { linha de ação do plano de sustentabilidade/ambiental sobre gestão da água. } \\
\text { AG3: Realização de atividades de sensibilização e conscientização sobre a } \\
\text { economia de água no âmbito da própria universidade. }\end{array}$ \\
\hline
\end{tabular}

Continua...

Revbea, São Paulo, V. 16, № 6: 83-103, 2021. 
...continuação.

\begin{tabular}{|c|l|}
\hline Construto & \multicolumn{1}{|c|}{ Operacionalização } \\
\hline Mobilidade & $\begin{array}{l}\text { MO1: Desenvolvimento de ações para reduzir a necessidade de } \\
\text { deslocamento na universidade e inter campus ou realizada boa ordenação } \\
\text { de escalas e horários ou flexibilizar a jornada de trabalho, etc. } \\
\text { MO2: Existência de ações de controle de estacionamento: políticas de } \\
\text { redução; cobrança de taxas; ações corretivas de estacionamentos } \\
\text { indevidos; priorização de vagas, etc. } \\
\text { MO3: Realização de ações de sensibilização e participação da comunidade } \\
\text { universitária sobre mobilidade. }\end{array}$ \\
\hline Resíduos & $\begin{array}{l}\text { RE1: A gestão de resíduos (elétricos, eletrônicos, químicos, biológicos e } \\
\text { radioativos) inclui o manejo adequado. } \\
\text { RE2: São realizados processos de minimização, separação e manejo } \\
\text { adequado dos resíduos orgânicos provenientes de refeitórios, dormitórios e } \\
\text { poda dos jardins? } \\
\text { RE3: São realizados processos de minimização, coleta seletiva e manejo } \\
\text { adequado dos resíduos, incluindo resíduos em obra de construção? }\end{array}$ \\
\hline Contratação & $\begin{array}{l}\text { CR1: Existe um plano, documento ou manual com protocolos de atuação } \\
\text { para a introdução de critérios de sustentabilidade e justiça social na } \\
\text { contratação de obras, serviços ou abastecimento. } \\
\text { CR2: São promovidas contratações e compras que seguem critérios de } \\
\text { comércio justo e inclusão social (contratação de pessoas portadoras de } \\
\text { deficiências, etc.). } \\
\text { CR3: As contratações responsáveis têm prioridade na escolha. }\end{array}$ \\
\hline
\end{tabular}

Fonte: autoria própria

As definições dos construtos foram fundamentadas nas referências empíricas obtidas na revisão da literatura e, forneceram a base teórica para seleção e planejamento dos itens indicadores. A integração proposta (sustentabilidade nas IES) visa posteriormente a criação de um instrumento de medida validado, determinado da seguinte maneira:

- Construto Políticas de Sustentabilidade (PS) $\rightarrow$ medido pelas variáveis PS1 a PS3;

- Construto Conscientização e Participação $(\mathrm{CP}) \rightarrow$ medido pelas variáveis CP1 a CP3;

- Construto Responsabilidade Socioambiental $(\mathrm{RS}) \rightarrow$ medido pelas variáveis RS1 a RS3;

- Construto Docência (DO) $\rightarrow$ medido pelas variáveis DO1 a DO3;

- Construto Energia (EN) $\rightarrow$ medido pelas variáveis EN1 a EN3;

- Construto Água (AG) $\rightarrow$ medido pelas variáveis AG1 a AG3;

- Construto Mobilidade (MO) $\rightarrow$ medido pelas variáveis MO1 a MO3;

- Construto Resíduos (RE) $\rightarrow$ medido pelas variáveis RE1 a RE3; e

- Contratação Responsável $(\mathrm{CR}) \rightarrow$ medido pelas variáveis CR1 a CR3. 
Um diagrama visual deste modelo, utilizando os 27 indicadores identificados, com os termos de erros, foi desenvolvido no software AMoS Graphics 24 ${ }^{\mathrm{TM}}$ (Analysis of Moment Structures) e é exibido na Figura 1.

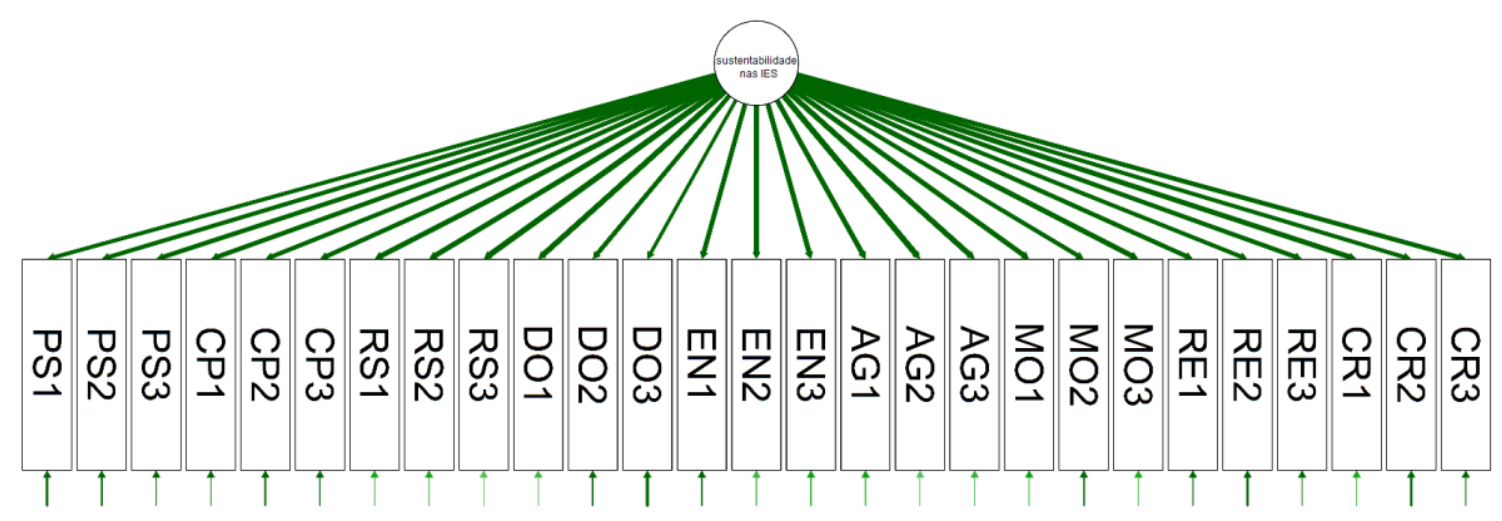

Figura 1: Modelo de mensuração

Fonte: autoria própria

Para estabelecimento da validade do instrumento se faz necessário pesquisas futuras para realizar procedimentos estatísticos, a fim de analisar a qualidade de ajuste do modelo, bem como a evidência da validade dos construtos.

\section{Conclusão}

Este artigo visou integrar as dimensões da sustentabilidade com a atividade-fim das IES brasileiras. Tal apresentação reforçou que todas as partes do sistema universitário são críticas para alcançar uma mudança transformadora, que só pode ocorrer conectando todas as dimensões. Esse tipo de pensamento é fundamental para implementar uma ação ambientalmente sustentável em nível local, regional e escalas globais em curto, médio e intergeracional períodos de tempo.

Embora a sustentabilidade seja uma inovação dentro de algumas IES, se faz necessário incorporá-lo de forma adaptada. Caso contrário, o processo de incorporação enfrentará forte resistência de indivíduos, levando a conflitos desnecessários e mais, não levará a sustentabilidade de fato.

Entender como o meio ambiente funciona, operar com energia renovável, eliminar o conceito de desperdício e viver dentro dos limites dos sistemas naturais são cruciais. Os gestores das IES devem reconhecer que o trabalho em direção à sustentabilidade é uma necessidade no mundo atual, onde os processos econômicos estão rapidamente degradando os recursos naturais e humanos sobre os quais as sociedades são total e mutuamente interdependentes. Pois, as políticas e estratégias da IES devem ser projetadas para integrar de maneira holística em todo o sistema universitário.

A presente pesquisa procurou desenvolver um modelo teórico para integrar as dimensões da sustentabilidade à atividade-fim e gestão das IES. 
Embora a pesquisa atual faça contribuições significativas, ainda apresenta limitações. Tal contexto de um único país pode restringir a generalidade das conclusões. Para lidar com essa preocupação, trabalhos futuros precisam investigar melhor a interação entre regulamentações públicas e privadas.

Dessa forma, para continuidade das pesquisas, recomenda-se completar as etapas da Modelagem de Equações Estruturais (MEE), por meio de procedimentos estatísticos, e a aplicação prática da definição do modelo teórico proposto neste estudo.

Agradecimentos: À Coordenação de Aperfeiçoamento de Pessoal de Nível Superior (CAPES), pelo apoio financeiro à pesquisa.

\section{Referências}

ALEIXO, A.M.; AZEITEIRO, U.M.; LEAL, S. Toward sustainability through higher education: sustainable development incorporation into Portuguese higher education institutions. In: DAVIM, J.P.; LEAL FILHO, W. (Eds), Challenges in Higher Education for Sustainability, Springer: London, 2016.

ALONSO-ALMEIDA, M.M.; MARIMON, F.; CASANI, F.; RODRIGUEZPOMEDA, J. Diffusion of sustainability reporting universities: current situation and future perspectives. Journal of Cleaner Production, v.106, p.144-154, 2015.

AMARAL, L.P.; MARTINS, N.; GOUVEIA, J.B. Quest for a sustainable university: a review. International Journal of Sustainability in Higher Education, v.16, n.2, p.155-172, 2015

BANSAL, P. Evolving Sustainably: A Longitudinal Study of Corporate Sustainable Development. Strategic Management Journal, v.26, p.197-218, 2005.

BEYNAGHI, A.; TRENCHER, G.; MOZTARZADEH, F.; MOZAFARI, M.; MAKNOON, R.; FILHO, W. L. Future Sustainability Scenarios for Universities: Moving beyond the United Nations Decade of Education for Sustainable Development. Journal of Cleaner Production, v.112, p.3464-3478, 2016.

BOFF, L. M; ORO, I. M; BEUREN, I.M. Gestão ambiental em Instituição de Ensino Superior na visão de seus dirigentes. Revista de Contabilidade da UFBA, Salvador, v.2, n.1, p. 4-13, 2008.

BOLZAN, L. M; WEBER, J. I; LÖBLER, M. L. Alinhamento ambiental em uma Instituição Pública de Ensino Superior. In: Encontro Internacional sobre Gestão Empresarial e Meio Ambiente. 12., 2010, São Paulo. Anais...São Paulo: FEA/USP, 2010.

COORDENAÇÃO DE APERFEIÇOAMENTO DE PESSOAL DE NÍVEL SUPERIOR (CAPES). Dados abertos da Pós-Graduação Stricto Sensu no 
Brasil 2017 a 2019, 2019. Disponível em: https://metadados.capes.gov.br/index.php/catalog/204. Acesso em: 11 mar. 2021.

CORTESE, A.D. The critical role of higher education in creating a sustainable future. Planning for Higher Education, v.31, p.15-22, 2003.

DISTERHEFT, A.; CAEIRO, S.; RAMOS, M.; AZEITEIRO, U.M. Environmental Management Systems (EMS) implementation processes and practices in European higher education institutions e top-down versus participatory approaches. Journal of Cleaner Production, v.31, p.80-90, 2012.

DISTERHEFT, A.; CAEIRO, S.; AZEITEIRO, U.M.; FILHO, W.L. Sustainability science and education for sustainable development in Universities: a way for transition, In: CAEIRO, S.; LEAL FILHO, W.; JABBOUR, C.; AZEITEIRO, U.M. (Eds), Sustainability Assessment Tools in Higher Education Institutions: Mapping Trends and Good Practices around the World, Springer International Publishing: Cardiff, 2013.

DISTERHEFT, A.; CAEIRO, S.S.; FILHO, W.L.; AZEITEIRO, U.M. The indicaremodel: measuring and caring about participation in higher education's sustainability assessment. Ecological Indicators, v.63, p.172-186, 2016.

DRAHEIN, A. D.; LIMA, E. P.; COSTA, S. E. G. Sustainability assessment of the service operations at seven higher education institutions in Brazil. Journal of Cleaner Production, v.212, p. 527-536, 2019.

DYER, G.; DYER, M. Strategic leadership for sustainability by higher education: the American College \& University Presidents' Climate Commitment. Journal of Cleaner Production, v.140, p.111-116, 2017.

DZIEDZIC, M; DZIEDZIC, R. Diagnóstico e proposta de redução de emissões Campus Universidade Positivo. In: Encontro Nacional sobre Gestão Empresarial e Meio Ambiente. 12., 2010, São Paulo. Anais... São Paulo: FEA/USP, 2010.

FIGUEIRÓ, P. S.; RAUFFLET, F. Sustainability in higher education: a systematic review with focus on management education. Journal of Cleaner Production, v.106, p. 22-23, 2015.

GODEMANN, J.; BEBBINGTON, J.; HERZIG, C.; MOON, J. Higher education and sustainable development: Exploring possibilities for organizational change. Accounting, Auditing \& Accountability Journal, v.27, n.2, p.218-233, 2014.

INSTITUTO NACIONAL DE METROLOGIA, QUALIDADE E TECNOLOGIA (INMETRO). Avaliação da Conformidade, 2021a. Disponível em: http://www.inmetro.gov.br/qualidade/certificacao.asp. Acesso em: 29 mar. 2021.

INSTITUTO NACIONAL DE METROLOGIA, QUALIDADE E TECNOLOGIA (INMETRO). Consulta às empresas certificadas, 2021b. Disponível em: https://certifiq.inmetro.gov.br/Consulta/ConsultaEmpresas. Acesso em: 29 mar. 2021. 
KARATZOGLOU, B. An in-depth literature review of the evolving roles and contributions of universities to education for sustainable development. Journal of Cleaner Production, v.49, p.44-53, 2013.

KOEHN, P.H.; UITTO, J.I. Universities and the Sustainable Development Future: Evaluating Higher-education Contributions to the 2030 Agenda. Routledge: New York, 2017.

LARRÁN JORGE, M.L.; MADUENO, J.H.; CEJAS, M.Y.C.; PEÑA, F. An approach to the implementation of sustainability practices in spanish universities. Journal of Cleaner Production, v.106, p.34-44, 2015.

LEAL FILHO, W.; MANOLAS, E.; PACE, P. The future we want: Key issues on sustainable development in higher education after Rio and the UN decade of education for sustainable development. International Journal of Sustainability in Higher Education, v.16, n.1, p.112-129, 2015.

LEMOS, P.F.I.; BRANDO, F. R.; ALMEIDA, P.; MÜLFARTH, R.C.K.; APRILANTI, T. M. G.; MARQUES, L. O.A.; JORGE, N.L.; MALHEIROS, T. F. The University of São Paulo on the 2017's GreenMetric Ranking. E3S Web Conf., v.48, 2018.

LIMA JR., F.R; OIKO, O. T; CAVICCHIOLI, F.F. Gestão de resíduos em universidade: Caracterização e análise da destinação de resíduos sólidos. In: Encontro Nacional sobre Gestão Empresarial e Meio Ambiente. 12., 2010, São Paulo. Anais...São Paulo: FEA/USP, 2010.

LOZANO, R. Incorporation and institutionalization of SD into universities: breaking through barriers to change. Journal of Cleaner Production, v.14, n.9-11, p.787-796, 2006.

LOZANO, R. Envisioning sustainability three-dimensionally. Journal of Cleaner Production, v.16, n.17, p.1838-1846, 2008.

LOZANO, R. Diffusion of sustainable development in universities curricula: an empirical example from Cardiff University. Journal Clean Production, v.18, n.7, p.637-644, 2010.

LOZANO, R. The state of sustainability reporting in universities. International Journal of Sustainability in Higher Education, v.12, n.1, p.67-78, 2011.

LOZANO, R.; CEULEMANS, K.; ALONSO-ALMEIDA, M.; HUISINGH, D.; LOZANO, F.J.; WAAS, T.; LAMBRECHTS, W.; LUKMAN, R.; HUG, J. A review of commitment and implementation of sustainable development in higher education: results from a worldwide survey. Journal of Cleaner Production, v.108, p.1-18, 2015.

LOZANO, R.; LOZANO, F.J.; MULDER, K.; HUISINGH, D.; WAAS, T. Advancing higher education for sustainable development: international insights and critical reflections. Journal of Cleaner Production, v.48, p.3-9, 2013a.

LOZANO, R.; LUKMAN, R.; LOZANO, F.J.; HUISINGH, D.; LAMBRECHTS, W. Declarations for sustainability in higher education: becoming better leaders,

revista brasileira educação ambiental 
through addressing the university system. Journal of Cleaner Production, v.48, p.10-19, $2013 b$.

MAXIMIANO JR., M. Indicadores brasileiros de extensão universitária. EDUFCG: Campina Grande, 2017.

MINISTÉRIO DA EDUCAÇÃO (MEC). Indicadores de Qualidade da Educação Superior, 2019. Disponível em: http://portal.inep.gov.br/web/guest/educacao-superior/indicadores-dequalidade/resultados. Acesso em: 21 mar. 2021.

MINISTÉRIO DO MEIO AMBIENTE (MMA). Curso de capacitação sustentabilidade na administração pública. Brasília, DF: MMA, 2017.

MOURA, B.A.; MOURA, L.B.A. Ranqueamento de universidades: reflexões acerca da construção de reconhecimento institucional [Ranking of universities: reflections on the construction of institutional recognition]. Acta Scientiarum. Education, v.35, n.2, p.213-222, 2013.

MUFF, K.; DYLLICK, T.; DREWELL, M.; NORTH, J.; SHRIVASTAVA, P.; HAERTLE, J. Management Education for the World: a Vision for Business Schools Serving People and Planet. Edward Elgar Publishing Limited, 2013.

OECD (ORGANISATION FOR ECONOMIC CO-OPERATION AND DEVELOPMENT). Reviews of National Policies for Education, OECD Publishing: Paris, 2018.

POPESCU, M.; BELEAU, C. Improving management of sustainable development in universities. Bulletin of the Transilvania University of Braşov, v.l, n.7, p.97-106, 2014.

ROHWEDER, L. Integrating environmental education into business schools'educational plans in Finland. GeoJournal, v.60, n.2, p.175-181, 2004.

RUSINKO, C. A. Using quality management as a bridge in educating for sustainability in a business school. International Journal of Sustainability in Higher Education, v.6, n.4, p.340-350, 2005.

SAMMALISTO, K.; SUNDSTROM, A.; HOLM, T. Implementation of sustainability in universities as perceived by faculty and staff is a model from a Swedish university. Journal of Cleaner Production, v.106, p.45-54, 2015.

SHAHIN, A.E.; MAHBOD, M.A. Prioritization of key performance indicators: An integration of analytical hierarchy process and goal setting. International Journal of Operations and Production Management, v.56, p.226-240, 2007.

SHARMA, S.; VREDENBURG, H. Estratégia ambiental corporativa proativa e desenvolvimento de capacidades organizacionais competitivas e valiosas. Revista de Gestão Estratégica, v.9, n.8, p.729-753, 1998.

SHARMA, S.; VREDENBURG, H.; WESTLEY, D.F. Strategic bridging: A role for the multinational corporation in Third World development. Journal of Applied Behavioral Science, v.30, n.4, p.458-476, 1994. 
SHIN, J.; TOUTKOUSHIAN, R. The past, present, and future of university ranking. In: SHIN, R.T.; TEICHLER, E.U. (Eds), University Ranking: Theoretical Basis, Methodology and Impacts on Global Higher Education, Springe: Dordrecht, 2011.

SHRIBERG, M. Institutional assessment tools for sustainability in higher education: strengths, weaknesses, and implications for practice and theory. Higher Education Policy, v.15, n2,2, p. 153-167, 2002.

STEPHENS, J.C.; HERNANDEZ, M.E.; ROMÁN, M.; GRAHAM, A.C.; SCHOLZ, R. W. Higher education as a change agent for sustainability in different cultures and contexts. International Journal of Sustainability in Higher Education, v.9, n.3, p.317-338, 2008.

STOUGH, T.; CEULEMANS, K.; LAMBRECHTS, W.; CAPPUYNS, V. Assessing sustainability in higher education curricula: a critical reflection on validity issues. Journal of Cleaner Production, p. 1-11, 2017.

STUBBS, W.; COCKLIN, C. Teaching sustainability to business students: shifting mindsets. International Journal of Sustainability in Higher Education, v.9, n.3, p.206-221, 2008.

THOMPSON, G. P. New faces, new opportunities: the environmental movement goes to business school. Environment. Science and Policy for Sustainable Development, v.27, n.4, p.6-30, 1985.

VEIGA, J. E. Indicadores socioambientais: evolução e perspectivas. Revista de Economia Política, v.29, n.4, p. 421-435, 2009.

WAAS, T.; HUGÉ, J.; VERBRUGGEN, A.; WRIGHT, T. Sustainable development: a bird's eye view. Sustainability, v.3, n.12, p.1637-1661, 2011.

WHITE, M.A. Sustainability: I know it when I see it. Ecological Economics, v.86, p.213-217, 2013. 